\title{
A NOTE ON GUL'KO COMPACT SPACES
}

\author{
GARY GRUENHAGE
}

\begin{abstract}
It is known that every Eberlein compact space has a dense $G_{\delta}$ metrizable subset. We answer a question of $M$. Talagrand by showing that the same is true for the wider class of $\mathrm{Gul}^{\prime}$ ko compact spaces. We also show that $\mathrm{Gul}^{\prime}$ ko compact spaces satisfy a certain covering property hereditarily.
\end{abstract}

1. Introduction. In this note we prove that every Gul'ko compact space has a dense $G_{\delta}$ metrizable subspace. This extends the same result for Eberlein compact spaces obtained by Namioka [Na] and Benyamini, Rudin, and Wage [BRW], and answers a question first mentioned by Talagrand [T2], and also stated in [CN and $\mathbf{N}$ ]. In fact, we define a larger class of spaces, similar in spirit to Rosenthal's characterization of Eberlein compacts, show that every Gul'ko compact is in this class, and that every member of this class has a dense $G_{\delta}$ metrizable subspace. We end this note with a proof that Gul'ko compacts satisfy a certain weak covering property hereditarily. This has as a corollary that $\mathrm{Gul}^{\prime}$ ko compact spaces which do not contain discrete subsets of measurable cardinality are Radon, a result proven by Talagrand [T2] for a somewhat smaller class of spaces.

2. Definitions and background. A compact space $X$ is Eberlein compact if it is homeomorphic to a weakly compact subset of a Banach space. Recall that a space is $k$-analytic if it is the continuous image of a closed subset of the product of the irrationals $P$ with some compact space. Talagrand [T1] showed that the Banach space $C(X)$ is $k$-analytic in the weak topology whenever $X$ is Eberlein compact, and now a compact space $X$ which satisfies the conclusion of Talagrand's theorem is called a Talagrand compact space. Gul'ko $[\mathbf{G u}]$ and Vasak $[\mathbf{V}]$ showed that a classical theorem for Eberlein compact spaces carried over to the class of those compact spaces $X$ such that $C(X)$, in the weak topology, is countably determined, i.e. the continuous image of a closed subset of the product of some subset $P^{\prime}$ of $P$ and a compact space, and these are now called $G u l^{\prime} k o$ compact spaces. All of these spaces are in the class of Corson compact spaces, which are those compacts that can be embedded in a $\Sigma$-product of real lines (i.e., in $\left\{x \in R^{\Gamma}: x(\alpha)=0\right.$ for at most countably many $\alpha$ in $\Gamma\}$ ).

As mentioned in the Introduction, it is known that every Eberlein compact has a dense $G_{\delta}$ metrizable subset. An example of Todorcevic [To, Theorem 9.14] shows that there is a Corson compact space with no dense metrizable subset at all. Talagrand [T3] showed that every Talagrand compact has a dense $G_{\delta}$ set consisting of $G_{\delta}$ points and Argyros and Negrepontis [AN] showed that every CCC Gul'ko compact space is metrizable. Our result generalizes the latter two results (note

Received by the editors June 10, 1985 and, in revised form, April 11, 1986.

1980 Mathematics Subject Classification (1985 Revision). Primary 54H99; Secondary 54D18, 46B20.

The author was partially supported by NSF Grant MCS 8301932. 
that a compact CCC space with a dense $G_{\delta}$ metrizable subset is separable, and that separable Corson compacta are metrizable), and answers a question stated in $[\mathbf{C N}, \mathbf{N}$, and $\mathbf{T 2}]$.

It will be more convenient to work with the so-called "Rosenthal characterizations" of these spaces. Rosenthal [R] showed that a compact space $X$ is Eberlein compact if and only if $X$ has a $\sigma$-point-finite $T_{0}$-separating cover by open $F_{\sigma}$ 's (a collection is $T_{0}$-separating if given any two points in $X$, some member of the collection contains exactly one of them). If " $\sigma$-point-finite" is replaced by "pointcountable," we get a characterization of Corson compacts [MR]. A recent result of Sokolov [So] gives characterizations of Talagrand and Gul'ko compacts which are slightly more complicated but in the same spirit. ${ }^{1}$ First, a definition:

DEFINITION. A collection $U$ of subsets of $X$ is weakly $\sigma$-point-finite if $U=$ $\bigcup\left\{\mathcal{U}_{n}: n \in \omega\right\}$ so that, for each $x \in X, \mathcal{U}=\bigcup\left\{\mathcal{U}_{n}: \operatorname{ord}\left(x, \mathcal{U}_{n}\right)<\omega\right\}$.

THEOREM [So]. A compact space is Gul'ko compact if and only if it has a weakly $\sigma$-point-finite $T_{0}$-separating cover by open $F_{\sigma}$ 's.

3. Dense metrizable subsets. We will define a class of spaces wider than the class of Gul'ko compacts and prove that every member of this class has a dense $G_{\delta}$ metrizable subspace.

Let us call a $T_{0}$-separating cover $\mathcal{U}$ of $X \sigma$-distributively point-finite if $\mathcal{U}=$ $\bigcup\left\{\mathcal{U}_{n}: n \in \omega\right\}$ so that, for each pair of distinct points $x, y \in X$, there exists $n \in \omega$ such that

(i) there exists $U \in \mathcal{U}_{n}$ containing exactly one of $x$ and $y$;

(ii) $\operatorname{ord}\left(x, U_{n}\right)<\omega$ or $\operatorname{ord}\left(y, \mathcal{U}_{n}\right)<\omega$.

It is easy to check that every weakly $\sigma$-point-finite $T_{0}$-separating collection is $\sigma$ distributively point-finite.

THEOREM 1. Every compact space which admits a $\sigma$-distributively point-finite $T_{0}$-separating open cover has a dense $G_{\delta}$ metrizable subspace.

REMARK. There are spaces satisfying the hypotheses of Theorem 1 which are not Gul'ko compact or even Corson compact; see the remark at the end of the next section for more details. However, we do not know if this condition characterizes Gul'ko compacts within the class of Corson compacts.

ProOF OF THEOREM 1. Let $\mathcal{U}=\bigcup\left\{\mathcal{U}_{n}: n \in \omega\right\}$ be a $\sigma$-distributively pointfinite $T_{0}$-separating open cover of $X$. For each $m, n \in \omega$, let

$$
X(n, m)=\left\{x \in X: \operatorname{ord}\left(x, \mathcal{U}_{n}\right)<m\right\} .
$$

Note that each $X(n, m)$ is closed in $X$. Let $\{n(k), m(k): k \in \omega\}$ enumerate $\omega \times \omega$. Let $\mathcal{G}(-1)=\{X\}$. We inductively define $\mathcal{G}(k), k \in \omega$, satisfying the following conditions:

(i) $\mathcal{G}(k)$ is a disjoint collection of open sets;

(ii) $\bigcup \mathcal{G}(k)$ is dense in $X$;

(iii) if $G \in \mathcal{G}(k)$ and $G^{\prime} \in \mathcal{G}(k-1)$, then either $G \cap G^{\prime}=\varnothing$ or $G^{-} \subset G^{\prime}$;

\footnotetext{
${ }^{1}$ Our main result was originally proven using a different "Rosenthal characterization" of Gul'ko compacts due to Mercourakis [M, Theorem 3.3]. However, Sokolov's characterization seems slightly better suited to our purpose.
} 
(iv) if $G \in \mathcal{G}(k)$, then either $G \cap X(n(k), m(k))=\varnothing$, or

$$
G \subset \bigcap\left\{U \in \mathcal{U}_{n(k)}: U \cap G \neq \varnothing\right\} .
$$

To define $\mathcal{G}(k)$, for each $G \in \mathcal{G}(k-1)$ let $\mathcal{V}(G)$ be a maximal disjoint collection of open sets satisfying (iv) whose closures are contained in $G$. Then let $\mathcal{G}(k)=$ $\bigcup\{\mathcal{V}(G): G \in \mathcal{G}(k-1)\}$. Then (i)-(iv) are satisfied as long as $\bigcup \mathcal{V}(G)$ is dense in $G$. To see that this is true, suppose $O$ is an open subset of $G$ and $O \cap(\bigcup \mathcal{V}(G))=\varnothing$. Then $O \subset X(n(k), m(k))$, for otherwise there exist $O^{\prime} \subset O^{\prime-} \subset O \backslash X(n(k), m(k))$, contradicting the maximality of $\mathcal{V}(G)$. Choose $x \in O$ such that $\operatorname{ord}\left(x, \mathcal{U}_{n(k)}\right)$ is as large as possible. Then the set $O \cap\left(\bigcap\left\{U \in \mathcal{U}_{n(k)}: x \in U\right\}\right)$ satisfies the second condition in (iv), so again the maximality of $\mathcal{V}(G)$ is contradicted. This shows that $\cup \mathcal{V}(G)$ is dense in $G$, and so the construction of the $\mathcal{G}(k)$ 's is complete.

Let $Y=\bigcap\{\bigcup \mathcal{G}(k): k \in \omega\}$. Then $Y$ is a dense $G_{\delta}$. To show that $Y$ is metrizable, it suffices to show that if $G(0) \supset G(1) \supset \cdots$, with $G(n) \in \mathcal{G}(n)$, then $\bigcap\{G(n): n \in \omega\}$ is a single point (for then, since $G(n+1)^{-} \subset G(n)$, the $G(n)^{\prime}$ 's are a base at this point, and so $\{G \cap Y: G \in \mathcal{G}(n), n \in \omega\}$ is a $\sigma$-locally finite base for $Y)$. To this end, suppose $x, y \in \bigcap\{G(n): n \in \omega\}$. There exist $n^{\prime} \in \omega$ and $U^{\prime} \in U_{n^{\prime}}$ such that $U^{\prime}$ contains exactly one of $x$ and $y$, and ord $\left(x, U_{n^{\prime}}\right)$ or $\operatorname{ord}\left(y, U_{n^{\prime}}\right)$ is finite. Thus $\{x, y\} \cap X\left(n^{\prime}, m^{\prime}\right) \neq \varnothing$ for some $m^{\prime} \in \omega$. Let $k$ be such that $(n(k), m(k))=\left(n^{\prime}, m^{\prime}\right)$. Since $G(k) \cap X\left(n^{\prime}, m^{\prime}\right) \neq \varnothing$, we have

$$
G(k) \subset \bigcap\left\{U \in \mathcal{U}_{n^{\prime}}: U \cap G(k) \neq \varnothing\right\} \subset U^{\prime} .
$$

This contradicts $\{x, y\} \subset G(k)$ and $U^{\prime}$ meets only one of $x$ and $y$. The proof is now complete.

4. Covering properties. In this section, we show that Gul'ko compacts satisfy a certain weak covering property hereditarily, and relate this to known results for covering properties of Corson and Eberlein compacts.

Recall that a space $X$ is metalindelöf ( $\sigma$-metacompact) if every open cover of $X$ has a point-countable ( $\sigma$-point-finite) open refinement. A cover $\mathcal{U}$ of $X$ is a weak $\theta$-cover if $\mathcal{U}=\bigcup\left\{\mathcal{U}_{n}: n \in \omega\right\}$ such that if $x \in X$, then $0<\operatorname{ord}\left(x, U_{n}\right)<\omega$ for some $n \in \omega$. $X$ is weakly submetacompact if every open cover of $X$ has an open refinement which is a weak $\theta$-cover. (Weakly submetacompact spaces are known in some circles as weakly $\theta$-refinable spaces.)

Yakovlev [Y] showed that Corson (Eberlein) compact spaces are hereditarily metalindelöf ( $\sigma$-metacompact). The author [G1] characterized Corson (Eberlein) compacts as those compact spaces $X$ such that $X^{2}$ is hereditarily metalindelöf $(\sigma$ metacompact), or equivalently, such that $X^{2} \backslash \Delta$ is metalindelöf ( $\sigma$-metacompact), where $\Delta=\{(x, x): x \in X\}$ is the diagonal. In [G2], we gave an example of a Corson compact space which is not hereditarily weakly submetacompact. We now show that Gul'ko compacts are hereditarily weakly submetacompact. In fact, we define a stronger covering property corresponding to Sokolov's characterization of Gul'ko compacts, and show that Gul'ko compacts have this covering property hereditarily. Let us call a space weakly $\sigma$-metacompact if every open cover has a weakly $\sigma$-point-finite open refinement. This covering property lies strictly between $\sigma$-metacompact and weakly submetacompact. 
THEOREM 2. Every Gul'ko compact space is hereditarily weakly $\sigma$-metacompact.

Before proving Theorem 2, we establish some technical lemmas.

LEMMA 3. If $U$ is a weakly $\sigma$-point-finite $T_{0}$-separating cover of $X$ by open $F_{\sigma}$ 's, then there exists a weakly $\sigma$-point-finite $T_{0}$-separating cover $U^{\prime}$ such that:

(i) each $U \in \mathcal{U}^{\prime}$ is a regular open set;

(ii) whenever $x \neq y \in X$, there exists $U \in U^{\prime}$ such that $U$ and $U^{-}$contain exactly one of $x$ and $y$.

ProOF. Write each $U \in \mathcal{U}$ as the union of regular open sets $U_{n}, n \in \omega$, whose closures are contained in $U$, and let $\mathcal{U}^{\prime}=\left\{U_{n}: n \in \omega, U \in \mathcal{U}\right\}$. It is easy to check that $U^{\prime}$ satisfies the desired conditions.

LEMMA 4. Let $\mathcal{U}$ be weakly $\sigma$-point-finite in $X$. Then $\mathcal{U}=\bigcup\left\{\mathcal{U}_{n}: n \in \omega\right\}$ such that:

(*) for each finite $\mathcal{V} \subset \mathcal{U}$ and each finite $F \subset X$, there exists $n \in \omega$ with $\mathcal{V} \subset \mathcal{U}_{n}$ and $\operatorname{ord}\left(F, U_{n}\right)$ finite.

ProOF. Let $\mathcal{U}=\bigcup\{\mathcal{U}(n): n \in \omega\}$ satisfy the weakly $\sigma$-point-finite condition. If $A$ is a finite subset of $\omega$, let $\mathcal{U}(A)=\bigcup\{U(n): n \in A\}$. We show that a reindexing of

$$
\left\{\mathcal{U}\left(A_{1}\right) \cap \mathcal{U}\left(A_{2}\right) \cap \cdots \cap \mathcal{U}\left(A_{n}\right): A_{i} \subset \omega, A_{i} \text { finite, } n \in \omega\right\}
$$

satisfies property $(*)$.

To this end, suppose $\mathcal{V}$ is a finite subset of $\mathcal{U}$ and $F$ is a finite subset of $X$. For each $x \in F$ and each $V \in \mathcal{V}$, there exists $n(x, V) \in \omega$ with $V \in \mathcal{U}(n(x, V))$ and $\operatorname{ord}(x, \mathcal{U}(n(x, V)))$ finite. Let $A(x)=\{n(x, V): V \in \mathcal{V}\}$. Note that $\mathcal{V} \subset \mathcal{U}(A(x))$ and $\operatorname{ord}(x, \mathcal{U}(A(x)))$ is finite. Thus $\bigcap\{\mathcal{U}(A(x)): x \in F\}$ witnesses satisfaction of $(*)$.

ProOF OF TheOREM 2. Let $X$ be Gul'ko compact. Then $X$ has a weakly $\sigma$-point-finite $T_{0}$-separating cover $\mathcal{U}$ by open $F_{\sigma}$ 's. We can assume that $\mathcal{U}$ satisfies conditions (i) and (ii) of Lemma 3, and $\mathcal{U}=\bigcup\{U(n): n \in \omega\}$ satisfies condition (*) of Lemma 4.

Let $O$ be an open subspace of $X$. It suffices to show that $O$ is weakly $\sigma$ metacompact, and for this it suffices to show that $O$ has a weakly $\sigma$-point-finite cover by open sets with compact closures (contained in $O$ ).

Let $H=X \backslash O$, and let $\mathcal{U}(n)^{+}=\mathcal{U}(n) \cup\left\{X \backslash U^{-}: U \in \mathcal{U}(n)\right\}$. Call a cover $\mathcal{V}$ of $H n$-minimal if

(i) $\mathcal{V} \subset \mathcal{U}(n)^{+}$;

(ii) for each $V \in \mathcal{V}$, there exists $y \in V \cap H \backslash \bigcup(\mathcal{V} \backslash\{V\})$ such that $\operatorname{ord}(y, \mathcal{U}(n))$ is finite.

Let

$$
\mathcal{W}(n, m)=\left\{\bigcap\left\{X \backslash V^{-}: V \in \mathcal{V}\right\}: \mathcal{V} \text { is an } N \text {-minimal cover of } H,|\mathcal{V}|<m\right\} \text {. }
$$

It remains to show:

Claim. $\mathcal{W}=\bigcup\{\mathcal{W}(n, m): n, m \in \omega\}$ is a weakly $\sigma$-point-finite cover of $O$.

Fix $x \in O$ and $W=\bigcap\left\{X \backslash V^{-}: V \in \mathcal{V}_{1}\right\}$ in $W$. We show that there exist $n, m \in \omega$ with $\operatorname{ord}(x, \mathcal{W}(n, m))<\omega$ and $W \in \mathcal{W}(n, m)$. For each $y \in H$, there exists $U(y) \in \mathcal{U}$ with $y \in U(y)$ and $x \notin U(y)^{-}$, or vice-versa. Let $U(y)^{*}$ denote 
the member of $\left\{U(y), X \backslash U(y)^{-}\right\}$which contains $y$. Let $\mathcal{V}_{2} \subset\left\{U(y)^{*}: y \in H\right\}$ be a finite minimal cover of $H$. Pick points $y(V) \in V \cap H \backslash \bigcup\left\{V^{\prime} \in V_{2}: V^{\prime} \neq V\right\}$.

For each $V \in U(n)^{+}$, there is $U(V) \in U(n)$ such that $V=U(V)$ or $V=$ $X \backslash U(V)^{-}$. Let $\mathcal{V}=\left\{U(V): V \in \mathcal{V}_{1} \cup \mathcal{V}_{2}\right\}, F=\{x\} \cup\left\{y(V): V \in \mathcal{V}_{2}\right\}$, and let $n$ satisfty property $(*)$ of Lemma 4 with respect to this $\mathcal{V}$ and $F$.

Let $m=|\mathcal{V}|+1$. Note that $W$ is in $\mathcal{W}(n, m)$. We finish the proof by showing that $x$ is point-finite in $\mathcal{W}(n, m)$. Suppose on the contrary that for each $k \in \omega$, we have a distinct element $W(k)=\bigcap\left\{X \backslash V^{-}: V \in \mathcal{V}(k)\right\}$ in $\mathcal{W}(n, m)$ containing $x$. Since each $\mathcal{V}(k)$ has size $<m$, we can assume that the $\mathcal{V}(k)$ 's have the same cardinality and $\mathcal{V}(i) \cap \mathcal{V}(j)=R$ whenever $i \neq j$. Since $x$ is point-finite in $\mathcal{U}(n)$, we can assume that $V=U(V)$ for each $V \in \mathcal{V}(k) \backslash R$. (This is where the fact that $\mathcal{U}$ consists of regular open sets is used.) Pick $V \in \mathcal{V}(0) \backslash R$, and $y(V) \in V$ satisfying condition (ii) in the definition of $n$-minimal. Then $y(V) \notin \bigcup R$, so for each $k, y(V) \in V(k)$ for some $V(k) \in \mathcal{V}(k) \backslash R$. But this contradicts ord $(y(V), \mathcal{U}(n))<\omega$.

REMARKS. (1) Let $(*)$ be the property that a space contains no discrete subspace of measurable cardinality. Schachermeyer $[\mathbf{S}]$ showed that every Eberlein compact space $X$ satisfying (*) is a Radon space, i.e., every Borel measure on $X$ is inner regular for compact sets, and Talagrand [T2] proved the same result for Talagrand compact spaces. Gardner $[\mathbf{G a}]$ showed that every compact hereditarily weakly submetacompact space satisfying $(*)$ is Radon; hence as a corollary to Theorem 2, we have that every Gul'ko compact satisfying $(*)$ is Radon.

(2) In view of the results of the author mentioned at the beginning of this section, it is natural to ask whether the condition " $X$ is compact and $X^{2}$ is hereditarily weakly $\sigma$-metacompact" characterizes Gul'ko compacts. We conjecture that it does. The weaker condition " $X$ is compact and $X^{2}$ is hereditarily weakly submetacompact" does not, because there are spaces satisfying this condition which are not even Corson compact (but we do not know if this condition characterizes Gul'ko compacts within the class of Corson compacts). To get an example, one can take $X$ to be the one-point compactification of any locally compact, nonmetrizable Moore space (such as Gillman and Jerison's space $\Psi[\mathbf{G J}]$ ). Then $X$ is not hereditarily metalindelöf, hence not Corson compact, but is follows from the fact that Moore spaces are subparacompact (and hence also weakly submetacompact) that $X^{2}$ is hereditarily weakly submetacompact. It is not difficult to check that this $X$ also has a $\sigma$-distributively point-finite $T_{0}$-separating cover by open $F_{\sigma}$ 's.

\section{REFERENCES}

[AN] S. Argyros and S. Negrepontis, On weakly $K$-countable determined spaces of continuous functions, Proc. Amer. Math. Soc. 87 (1983), 731-736.

[BRW] Y. Benyamini, M. E. Rudin, and M. Wage, Continuous images of weakly compact subsets of Banach spaces, Pacific J. Math. 70 (1977), 309-324.

[CN] W. W. Comfort and S. Negrepontis, Chain conditions in topology, Cambridge Tracts in Math., Vol. 79, Cambridge Univ. Press, Cambridge, 1982.

[Ga] R. J. Gardner, The regularity of Borel measures and Borel measure compactness, Proc. London Math. Soc. (3) 30 (1975), 95-113.

[GJ] L. Gillman and M. Jerison, Rings of continuous functions, University Series in Higher Math., Van Nostrand, Princeton, N.J., 1960.

[G1] G. Gruenhage, Covering properties on $X^{2} \backslash \Delta, W$-sets, and compact subsets of $\Sigma$-products, Topology Appl. 17 (1984), 287-304.

[G2] __ On a Corson compact space of Todorcevic (to appear). 
[Gu] S. P. Gul'ko, On the structure of spaces of continuous funtions and their complete paracompactness, Russian Math. Surveys 34 (1979), 36-44 (=Uspekhi Math. Nauk 34 (1979), 33-40).

[M] S. Mercourakis, On weakty conntably determined Banach spaces, preprint.

[MR] E. Michael and M. E. Rudin, A note on Eberlein compacts, Pacific J. Math. 72 (1977), 487-495.

[N] S. Negrepontis, Banach spaces and topology, Handbook of Set-Theoretic Topology (K. Kunen and J. E. Vaughan, eds.), North-Holland, Amsterdam, 1984.

[Na] I. Namioka, Separate and joint continuity, Pacific J. Math. 51 (1974), 515-531.

[R] H. P. Rosenthal, The heredity problem for weakly compactly generated Banach spaces, Compositio Math. 28 (1974), 83-111.

[S] W. Schachermeyer, Eberlein-compacts et spaces de Radon, C. R. Acad. Sci. Paris Ser. A 284 (1977), 405-407.

[So] G. A. Sokolov, On some classes of compact spaces lying in $\Sigma$-products, Comm. Math. Univ. Carolin. 25 (1984), 219-231.

[T1] M. Talagrand, Sur une conjecture de H. H. Corson, Bull. Sci. Math. 99 (1975), 211-212.

[T2] _ Espaces de Banach faiblement K-analytiques, Ann. of Math. (2) 110 (1979), 407-438.

[T3] _ Deux generalisations d'un theoreme de I. Namioka, Pacific J. Math. 81 (1979), 239-251.

[To] S. Todorcevic, Thees and linearly ondered sets, Handbook of Set-Theoretic Topologyy (K. Kunen and J. E. Vaughan, eds.), North-Holland, Amsterdam, 1984.

[V] L. Vasak, On one generalization of weakdy compactly generated Banach spaces, Studai Math. 70 (1981), 11-19.

[Y] N. N. Yakovlev, On bicompacta in $\Sigma$-products and related spaces, Comm. Math. Univ. Carolin. 21 (1980), 263-282.

Division of Mathematics, Auburn University, Auburn, Alabama 36849 